\title{
Syllable cueing and segmental overlap effects in tip-of-the-tongue resolution
}

Nina Jeanette Sauer

Goethe-University Frankfurt, Phorms Education Frankfurt

https://doi.org/10.36505/ExLing-2016/07/0035/000294

\begin{abstract}
The tip-of-the-tongue (TOT) phenomenon refers to a temporary word finding failure. To induce TOTs in the lab, a common method is to ask for terms after providing created definitions. When in a TOT, syllable cues were presented in order to manipulate TOT resolution. After the presentation of the correct first syllable of the target word, TOTs could be resolved faster and more accurately than after the presentation of an incorrect syllable of some other word or the control condition (Experiment 1: syllable cueing effect). The presentation of the extended syllable of the word (the first syllable with one more segment) facilitated TOT resolution and boosted lexical retrieval even more than the regular syllable (Experiment 2: segmental overlap effect). Key words: tip-of-the-tongue (TOT), resolution, cueing, syllable, segmental overlap
\end{abstract}

\section{Introduction}

The tip-of-the-tongue phenomenon (TOT) represents a temporary impairment in speech production. When experiencing a TOT, one has access to semantic (concept) and syntactic information (lemma) but only partial access to phonological information (lexeme). While the complete word form cannot be retrieved, one has a strong feeling of knowing the word and "recall is felt to be imminent" (Brown \& McNeill 1966, p. 325). Often, speakers are able to retrieve the first letter or phoneme, the number of syllables and also words with similar sound and similar meaning (Brown 2012, p. 196).

In order to induce TOTs in a laboratory setting, definitions were presented on a computer screen, for example, "a lift consisting of a series of linked compartments moving continuously" for paternoster. In the cueing paradigm so far, syllable cues were embedded in words or pseudowords, and presented in word lists in order to manipulate TOT resolution (for an overview, see Hofferberth-Sauer \& Abrams 2014). Abrams, White, and Eitel (2003) illustrated, for example, that the entire first syllable is required for TOT resolution - the first phoneme or first grapheme alone had no effect. In the present studies, syllable cues were presented in isolation. The advantage of this procedure is that the syllable itself has no semantic and syntactic information. The

ExLing 2016: Proceedings of $7^{\text {th }}$ Tutorial and Research Workshop on Experimental Linguistics, 27 June - 2 July 2016, Saint Petersburg, Russia 
156 N.J. Sauer

presentation of isolated correct, incorrect, and extended syllables is new in TOT research.

\section{Previous studies}

In the pre-tests, definitions had been collected and verified (Hofferberth, 2011). In two pilot studies (Hofferberth, 2012), the design of the experiment was evaluated, and more definitions were collected and validated. Thereafter, two experiments were performed. The first experiment (Hofferberth 2014; Hofferberth-Sauer \& Abrams 2014) will be presented here only marginally while the focus is on the second experiment (cf. 3.). All the data was collected within my Ph.D. project (Sauer 2015).

\section{Experiment 1}

In the first experiment, definitions were presented on a computer screen. When in a TOT, one of three cues was presented. It was shown that after the presentation of the correct syllable (e.g., pa for paternoster), TOTs could be resolved about twice as fast compared to after an incorrect syllable (e.g., $c o)$ and to the control condition $(x \times x)$. The correct syllable also led to significantly more accurate answers $(M=73.5 \%, S D$ $=18.6 \%)$ compared to the control condition $(M=24.3 \%, S D=16.4 \%$, $t(47)=16.39, p<.001)$, and to the incorrect syllable $(M=16.0 \%, S D=$ $13.6 \%, t(47)=20.06, p<.001)$. The control condition led to significantly more accurate TOT resolutions compared to the incorrect syllable $(t(47)$ $=3.71, p=.001)$. The incorrect syllable did not block TOT resolution (not leading to more inaccurate answers), but there was an inhibition effect: There were fewer accurate answers and more unresolved TOTs. After demonstrating the cueing effect of the first syllable in Experiment 1 , a further experiment was conducted in order to test if the syllable border plays a role (syllable preference effect).

\section{Experiment 2}

Method

\section{Participants}

69 under- and postgraduates (42 female, 27 male) between 21 and 35 years $(M=27.9$ years, $S D=4.3)$ participated in this study.

\section{Apparatus and material}

The material was visually presented on a computer screen using the program Presentation. There were 240 definitions of German nouns 
presented in order to induce TOTs (the English examples here are only for demonstration purposes).

\section{Procedure}

The subjects were told to press a button on the keyboard as fast as possible indicating that they know the word (KNOW), that they do not know the word (DON'T KNOW), or that the word is on their tip of the tongue (TOT). They had 10 seconds to react to the definition. After pressing KNOW, they typed in the answer, and another definition was presented. After pressing DON'T KNOW, the next definition appeared on the screen. After pressing TOT, a cue was presented visually: either the regular syllable (e.g., $p a$ for paternoster), the extended syllable (e.g., pat), or the control condition (marked by $x \times x$ ). The cue was presented for 25 seconds. In this time, the subjects had to type in their answer.

\section{Results TOT rate}

The number of TOTs varied between $21(8.8 \%)$ and 194 TOTs $(80.8 \%)$. Through 16560 stimuli overall, 5600 TOTs were induced, i.e., the TOT rate was $33.8 \%$ with 81 TOTs per person on average $(S D=14.7 \%)$. Out of the 5600 TOTs, 3385 TOTs $(60.5 \%)$ were resolved in the given time of 25 seconds, with reaction times (RTs) between $571 \mathrm{~ms}$ and $24948 \mathrm{~ms}$ $(M=4049 \mathrm{~ms}, S D=4325 \mathrm{~ms})$. There were $50.3 \%$ accurate answers, and $10.2 \%$ inaccurate answers.

\section{Cue analysis}

The number of accurate TOT resolutions differed between the three types of cues $(F(2,136)=415.65, p<.001)$. With the extended syllable, TOTs were accurately resolved significantly more often $(M=72.0 \%, S D=18.7 \%)$ in comparison to the regular syllable $(M=60.3 \%, S D=19.0 \%, t(68)=7.00, p<$ $.001)$, and to the control condition $(M=18.7 \%, S D=13.0 \%, t(68)=26.26, p<$ $.001)$. The regular syllable led to significantly more accurately resolved TOTs $(t(68)=19.80, p<.001)$.

The RTs were significantly shorter after the presentation of the extended syllable $(M=2330 \mathrm{~ms}, S D=887 \mathrm{~ms})$ in comparison to the regular syllable $(M=2803 \mathrm{~ms}, S D=1166 \mathrm{~ms}, t(67)=3.92, p<.001)$, and to the control condition $(M=3017 \mathrm{~ms}, S D=1592 \mathrm{~ms}, t(62)=2.89$, $p=.005)$. There was no significant difference between the regular syllable and the control condition $(t(62)=0.78, p=.436)$. 


\section{Discussion}

While Experiment 1 showed the syllable cueing effect, i.e., the correct first syllable helped to overcome transmission deficits from the lemma to the lexeme level, Experiment 2 showed the segmental overlap effect, i.e. a speaker needs even more than the first syllable for successful TOT resolution. It was demonstrated that the extended syllable (e.g., pat for paternoster) significantly speeded up lexical access (shorter RTs), and significantly increased TOT resolution (more accurate answers) compared to after the regular syllable (e.g., $p a$ ) and to the control condition $(x \times x)$. The key factor was not the syllable per se but the information content: the bigger the segmental overlap between cue and target, the faster and better the TOT resolution. Therefore, it is helpful to get as much information as possible about the beginning of the target word. The unit of the syllable only plays a marginal role.

Syllable cueing and segmental overlap effects do not have to exclude each other but rather can both be explained within speech production models that allow for an interactive activation spreading and have a syllable level below the phoneme level. For an interpretation and discussion of these results within different models of speech production see Sauer and Schade (2016).

\section{References}

Abrams, L., White, K.K., Eitel, S.L. 2003. Isolating phonological components that increase tip-of-the-tongue resolution. Memory \& Cognition, 31, 1153-1162.

Brown, A.S. 2012. The tip of the tongue state. New York, Psychology Press.

Brown, R., McNeill, D. 1966. The "tip of the tongue" phenomenon. Journal of Verbal Learning and Verbal Behaviour, 5, 325-337.

Hofferberth, N. J. 2011. The tip-of-the-tongue phenomenon: Search strategy and resolution during word finding difficulties. Proc. $4^{\text {th }}$ ISCA Tutorial and Research Workshop on Experimental Linguistics, ExLing 2011, 83-86. Paris, France.

Hofferberth, N. J. 2012. On the role of the syllable in tip-of-the-tongue states. Proc. International Conference of Experimental Linguistics, ExLing 2012, 57-60. Athens, Greece.

Hofferberth, N. J. 2014. Resolution of lexical retrieval failures. Reaction time data in the tip-of-the-tongue paradigm. Proceedings of the International Seminar on Speech Production. ISSP 05-08 May 2014, 194-197. Cologne, Germany.

Hofferberth-Sauer, N.J., Abrams, L. 2014. Resolving tip-of-the-tongue states with syllable cues. In Torrens, V. and Escobar, L. (eds.), The processing of lexicon and morphosyntax, 43-68. Newcastle, Cambridge Scholars Publishing.

Sauer, N.J. 2015. Das Tip-of-the-Tongue-Phänomen. Zur Rolle der Silbe beim Auflösen von Wortfindungsstörungen. Doctoral dissertation, Frankfurt am Main, Johann Wolfgang Goethe-Universität. doi: 10.13140/RG.2.1.1229.8645

Sauer, N. J. and Schade, U. 2016. Über die Entstehung und Auflösung von Versprechern und Tip-of-the-Tongue-Zuständen. Manuscript in preparation. 